\title{
Preface
}

The cryosphere describes environments where water appears in a frozen form. It includes permafrost, glaciers, ice sheets, and sea ice and is currently more affected by Global Change than most other regions of the Earth. In the cryosphere, limited water availability and subzero temperatures cause extreme conditions for all kinds of life, but many microorganisms can cope with this extremely well. The cryosphere's microbiota displays an unexpectedly large genetic reservoir and taxonomic as well as functional diversity, which, however, we are still only beginning to map. Moreover, microbial communities influence the response mechanisms of the cryosphere towards Global Change. Altered patterns of seasonal temperatures and precipitation are already occurring in many cryotic ecosystems, affecting microbial element cycling, adaptation strategies, and physiology. Activation of nutrients by thawing permafrost and increasing thaw layer thickness, as well as erosion, for example, renders nutrient stocks accessible to microbial activities. Also, glacier melt and retreat stimulate microbial life, which in turn influences albedo and surface temperatures. In this context, the functional resilience of microbial communities in the cryosphere is of major interest and concern. Particularly important is the ability of microorganisms, and microbial communities as a whole, to respond to changes in their surroundings by intracellular regulation and population shifts within functional and ecological niches, respectively.

Research on microbial life exposed to permanent freeze or seasonal freeze-thaw cycles has led to astonishing findings about microbial versatility, adaptation, and diversity. Microorganisms thrive in cold habitats and new sequencing techniques have produced large amounts of genomic, metagenomic, and metatranscriptomic data that allow insights into the fascinating microbial ecology and physiology at low and subzero temperatures. Moreover, some of the frozen ecosystems such as permafrost in Siberia, Canada, and Alaska constitute major global carbon and nitrogen storages but can also act as sources of the greenhouse gases methane and nitrous oxide impacting our climate globally.

We hope that with this book, you find exciting insights into the life strategies of single microbial species and the entire microbiome in prominent ecosystems of the cryosphere. You will learn about microbial climate feedbacks and multiple response mechanisms to environmental change and how this potentially changes biogeochemical cycles of the cryosphere and beyond.

Susanne Liebner and Lars Ganzert 



\section{Volume published in the series}

\section{Volume 1}

Jens Kallmeyer, Dirk Wagner (Eds.)

Micobial Life of the Deep Biosphere

ISBN 978-3-11-030009-3

\section{Volume 2}

Corien Bakermans (Ed.)

Micobial Evolution under Extreme Conditions

ISBN 978-3-11-033506-4

\section{Volume 3}

Annette Summers Engel (Ed.)

Micobial Life of Cave Systems

ISBN 978-3-11-033499-9

\section{Volume 4}

Blaire Steven (Ed.)

The Biology of Arid Soils

ISBN 978-3-11-041998-6

\section{Volume 5}

Jens Kallmeyer (Ed.)

Life at Vents and Seeps

ISBN 978-3-11-049475-4

\section{Volume 6}

Natuschka Lee (Ed.)

Biotechnological Applications of Extremophilic Microorganisms

978-3-11-042773-8

\section{Volume 8}

Étienne Yergeau

Advanced Techniques for Studying Microorganisms in Extreme Environments ISBN: 978-3-11-052464-2 
